\title{
DISTENSÃO ABDOMINAL AGUDA EM PACIENTE COM SÍNDROME CONSUMPTIVA
}

\section{ABDOMINAL DISTENSION IN PATIENT WITH WASTING SYNDROME}

Alfredo Hümmelgen Junior1, Eiji Rafael Nakahashi1, Isadora Brondani Ceolin², Vivien Ritchie Bittencourt² e Maurício de Carvalho ${ }^{3}$

Caso Clínico: Uma mulher de 66 anos, com antecedente de perda não intencional de $20 \mathrm{~kg}$ nos últimos 2 anos, foi admitida na enfermaria com história de dor e aumento de volume abdominal há 15 dias, eliminando flatos e com evacuação normal. A paciente, então, evoluiu com náuseas, vômitos, piora da dor e distensão. Uma TC de abdome demonstrou dilatação de todo o cólon, ausência de pontos de obstrução e diâmetro cecal de $10 \mathrm{~cm}$, além de sinais de carcinomatose peritoneal e massa em anexos uterinos. Foi submetida a tratamento com colonoscopia descompressiva, resultando na melhora temporária dos sintomas.
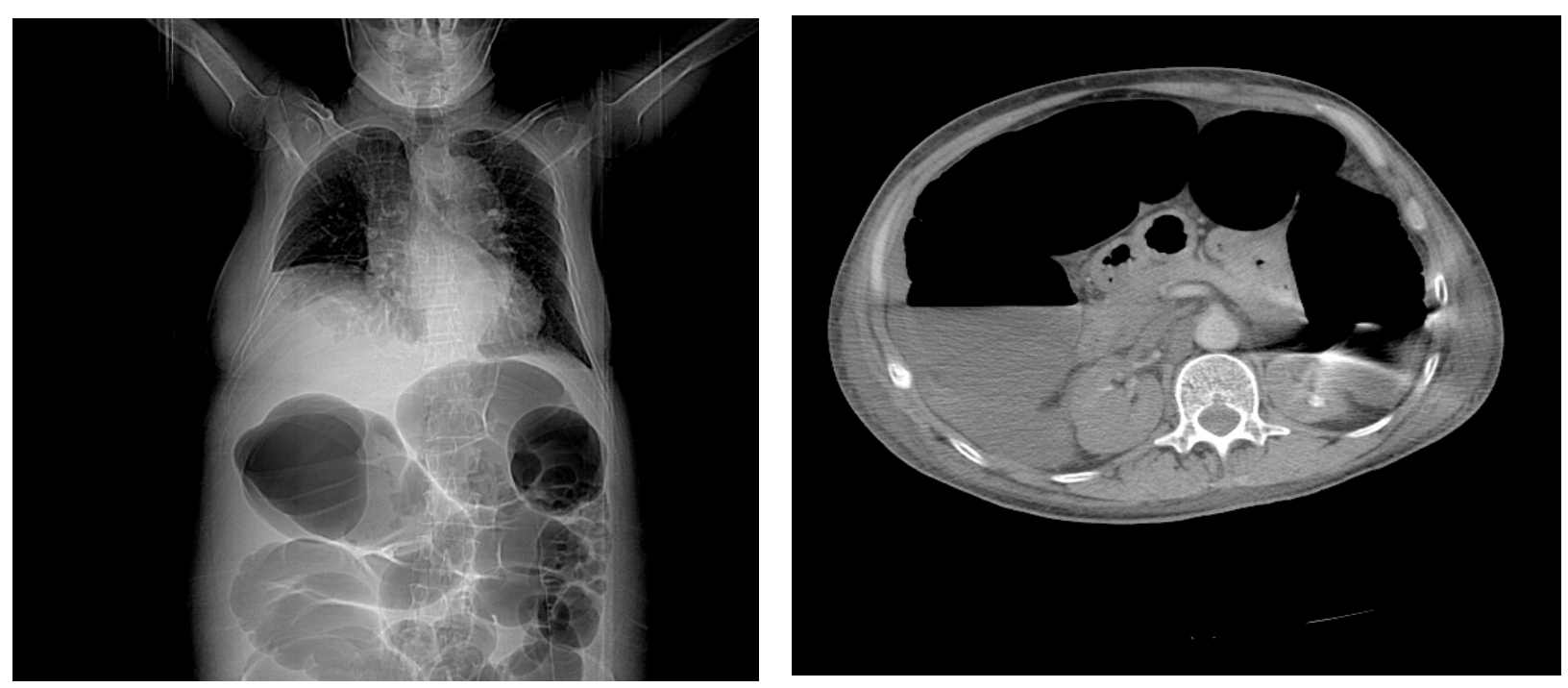

Figura 1 e 2: Antes da desobstrução em plano longitudinal e transversal, respectivamente.

Qual o provável diagnóstico?
A. Suboclusão intestinal por carcinomatose
B. Megacólon chagásico
C. Síndrome de Ogilvie
D. Isquemia mesentérica
E. Volvo de sigmóide

\footnotetext{
1 - Acadêmicos de Medicina - Universidade Federal do Paraná (HC-UFPR)

2- Médicos Residentes de Clínica Médica, HC-UFPR.

3- Chefe do Departamento de Clínica Médica, HC-UFPR. Professor de Nefrologia da
} Pontíficia Universidade Católica do Paraná (PUC-PR). 


\section{RESPOSTA C - Síndrome de Olgivie (pseudobstrução colônica aguda)}

A pseudo obstrução colônica aguda, também denominada Síndrome de Ogilvie, é caracterizada por uma intensa dilatação do ceco e do cólon direito, podendo ainda estender-se para o reto, sem que haja uma causa obstrutiva de natureza mecânica para explicar a parada do trânsito intestinal1-4. A patogênese exata da dilatação do colón é ainda desconhecida, porém acredita-se que resulte de um desequilíbrio entre sistema nervoso autônomo simpático e parassimpático ${ }^{1}$. Pode estar relacionada com outras condições clínicas, como traumas, cirurgias, infarto agudo do miocárdio, doenças neurológicas e doenças retroperitoneais (neoplasias ou hemorragias). Pode ainda ser secundária ao uso de alguns medicamentos como opióides, bloqueadores de canal de cálcio, agonistas adrenérgicos e anestésicos utilizados em anestesia epidural ${ }^{4}$.

Apesar da síndrome caracteristicamente afetar mais homens e pessoas da faixa etária acima dos 60 anos, já existem casos reportados de crianças com a pseudo obstrução $0^{4,6}$. O principal sintoma em pacientes com pseudo obstrução colônica aguda é a distensão abdominal gradual, geralmente levando 3-7 dias, podendo ocorrer de maneira rápida em até 24 a 48 horas. Outros sintomas frequentes são dor abdominal associada a náuseas e vômitos, ocorrendo em mais da metade dos pacientes ${ }^{1,5}$. 0 risco de perfuração colônica aumenta quando a dilatação cecal ultrapassa 10 a $12 \mathrm{~cm}$ ou quando a dilatação perdura por mais de 6 dias, sendo a duração o quesito mais importante em relação ao risco de perfuração4,6.

O diagnóstico da síndrome de Ogilvie é um diagnóstico de exclusão. Primariamente, deve-se excluir as causas obstrutivas verdadeiras. Deve-se suspeitar do diagnóstico naqueles pacientes com distensão ou dor abdominal e com exame físico com abdome timpânico à percussão. 0 diagnóstico, porém, só é estabelecido através de exames de imagem. A colonoscopia não deve ser utilizada como ferramenta diagnóstica, uma vez que a insuflação com ar poderá distender ainda mais o colón, com risco de perfuração $0^{1,2,6}$. O enema opaco ou tomografia computadorizada de abdome podem ser realizados para a detecção de alguma obstrução mecânica. É importante ressaltar que a tomografia pode ainda detectar sinais de isquemia e perfurações, além de anormalidades em estruturas pericolônicas ${ }^{2}$. Os principais diagnósticos que devem ser incluídos como diagnósticos diferenciais são: Carcinoma, aderências, dilatação gástrica aguda, impactação fecal, volvo mesentérico, isquemias mesentéricas e megacólon chagásico ${ }^{3}$.

O tratamento inicial é suportivo, tendo como alvo o controle da dor, a nutrição ideal do paciente e o controle dos sinais de dismotilidade intestinal. 0 tratamento conservador consiste na descontinuação de certas medicações em uso pelo paciente, principalmente anticolinérgicos, tricíclicos, bloqueadores do canal de cálcio, drogas antiparkinsonianas e clonidina. Além disso, é importante corrigir os distúrbios hidroeletrolíticos do paciente. Pode haver necessidade de passagem de sonda nasogástrica para descompressão em alguns casos. 0 tratamento medicamentoso é feito com a neostigmina na dose de 2-2,5 mg intravenoso a cada 3 ou 4 horas. Se não houver resposta, pode-se repetir uma dose maior de neostigmina $(2,5-3 \mathrm{mg})^{7}$. Caso haja alguma contraindicação ao uso da neostigmina, tem-se como alternativa a realização de descompressão por colonoscopia, sendo esta manobra efetiva em $85 \%$ dos $\operatorname{casos}^{6,7}$. A descompressão percutânea e o tratamento cirúrgico devem ser reservados aos pacientes com refratariedade ao tratamento conservador ou aos casos mais graves, com perfuração ou isquemia ${ }^{4}$. Caso exista alguma dessas ultimas condições, o tratamento cirúrgico está indicado através de um tubo de cecostomia, se possível, ou mesmo uma colectomia, geralmente à direita6,7.

\section{REFERÊNCIAS}

1. Ribas-Filho JM, Malafaia O, Fouani NM, Fouani MM, Justen MS, Trevisan NB, Lopes AD, Lopes GL. Ogilvie's Syndrome (Acute pseudo-intestinal obstruction) - A case report. ABCD Arq Bras Cir Dig 2009; 22(2):124-6

2. Jain A., Vargas HD. Advances and Challenges in the Management of Acute Colonic Pseudo-Obstruction (Ogilvie's Syndrome). Clin Colon Rectal Surg. 2012; 25:37-45.

3. Vanek VW, Al-Sati M. Acute pseudo-obstruction of the colon (Ogilvie's Syndrome): an analysis of 400 cases. Dis Colon Rectum 1986; 29: 203-210

4. Oliveira L, Santos GR, Marques GL, Dunin FS, Zetola VHF, Carvalho M. The giant caecum with no apparent reason: Ogilvie's Syndrome. International Journal of Case Reports in Medicine 2014; 2014: 1-4

5. Stephenson BM, Morgan AR, Salaman JR, Wheeler $\mathrm{MH}$. Ogilvie's Syndrome: a new approach to an old problem. Dis Colon Rectum 1995; 38: 424-427

6. Camilleri M. Acute colonic pseudo-obstruction (Ogilvie's Syndrome). UpToDate. Mar 26,2015. Disponivel em: < http://www.uptodate.com >. Acesso em: 22/04/2016

7. Cagir B. Intestinal Pseudo-Obstruction Treatment and Management. Medscape. Dec 28, 2015. Disponível em:

http://emedicine.medscape.com/article/2162306treatment> . Acesso em 22/04/2016 